\title{
Utilização de impressoras 3D para o desenvolvimento de metodologias ativas em
}

\section{cursos de Engenharia}

\author{
Use of 3D printers for the development of active methodologies in Engineering courses \\ Uso de impresoras 3D para el desarrollo de metodologías activas en cursos de Ingeniería
}

Recebido: 24/07/2021 | Revisado: 29/07/2021 | Aceito: 02/08/2021 | Publicado: 08/08/2021

\author{
João Batista Mendes Barbosa \\ ORCID: https://orcid.org/0000-0002-9597-059X \\ Universidade Regional do Cariri, Brasil \\ E-mail: joao.mendes@urca.br \\ Francisca Jeane Sidrim de Souza Figueiredo Mendonça \\ ORCID: https://orcid.org/0000-0003-4979-2101 \\ Universidade Regional do Cariri, Brasil \\ E-mail: jeanne.sidrim@urca.br \\ Frederico Romel Maia Tavares \\ ORCID: https://orcid.org/0000-0001-5156-2167 \\ Universidade Regional do Cariri, Brasil \\ E-mail: prof_fred@hotmail.com \\ José Francisco de Sousa Figueredo \\ ORCID: https://orcid.org/0000-0003-2032-5198 \\ Universidade Regional do Cariri, Brasil \\ E-mail: jose.figueredo.urca.br \\ Maria Juliana Ferreira Leite \\ ORCID: https://orcid.org/0000-0002-1772-0878 \\ Universidade Regional do Cariri, Brasil \\ E-mail: juliana.ferreira@urca.br
}

\begin{abstract}
Resumo
Esta pesquisa teve como objetivo verificar a importância do uso da Manufatura Aditiva no ensino de Engenharia com o uso de Metodologias Ativas. O estudo apresenta os resultados da experiência dos alunos com o uso de Impressoras 3D em atividades de Metodologias Ativas. Suas experiências e percepções mostraram as implicações da aplicação dessas práticas em sala de aula. Foi realizado um estudo de caso para melhor compreender o fenômeno estudado. Também se realizou uma revisão bibliográfica de literatura para ter uma visão global do campo da pesquisa. Uma leitura seletiva foi necessária, os conteúdos foram analisados e um formulário online foi aplicado em formato de entrevista para coleta de dados. Foi possível verificar como o uso de Metodologias Ativas e Manufatura Aditiva se conecta e possibilita os projetos desenvolvidos pelos alunos garantindo aulas interativas e dinâmicas, onde o desenvolvimento do pensamento crítico e analítico é estimulado para a tomada de decisão. Está pesquisa pode ser utilizada como referência norteadora para professores e engenheiros perceberem a real importância das práticas de PBL com o uso de impressoras 3D, apresentadas neste estudo.
\end{abstract}

Palavras-chave: Metodologias ativas; Manufatura aditiva; Educação; Ensino para engenharia; Impressão 3D.

\begin{abstract}
I this research aimed to verify the importance of the use of Additive Manufacturing in engineering teaching with the use of Active Methodologies. The study presents the results of the students' experience with the use of 3D Printers in Active Methodologies activities. Their experiences and perceptions showed the implications of applying these practices in the classroom. A case study was conducted to better understand the phenomenon studied. A literature literature review was also conducted to get a global view of the field of research. A selective reading was required, the contents were analyzed and an online form was applied in interview format for data collection. It was possible to verify how the use of Active Methodologies and Additive Manufacturing connects and enables the projects developed by the students ensuring interactive and dynamic classes, where the development of critical and analytical thinking is stimulated for decision making. This research can be used as a guide for teachers and engineers to realize the real importance of PBL practices with the use of 3D printers, presented in this study.
\end{abstract}

Keywords: Active methodologies; Additive manufacturing; Education; Teaching for engineering; 3D printing.

\section{Resumen}

Esta investigación tuvo como objetivo verificar la importancia del uso de la Fabricación Aditiva en la enseñanza de la ingeniería con el uso de Metodologías Activas. El estudio presenta los resultados de la experiencia de los estudiantes 
con el uso de impresoras 3D en actividades de Metodologías Activas. Sus experiencias y percepciones mostraron las implicaciones de aplicar estas prácticas en el aula. Se realizó un estudio de caso para comprender mejor el fenómeno estudiado. También se llevó a cabo una revisión de la literatura para obtener una visión global del campo de la investigación. Se requirió una lectura selectiva, se analizaron los contenidos y se aplicó un formulario en línea en formato de entrevista para la recolección de datos. Fue posible verificar cómo el uso de Metodologías Activas y Fabricación Aditiva conecta y posibilita los proyectos desarrollados por los estudiantes asegurando clases interactivas y dinámicas, donde se estimula el desarrollo del pensamiento crítico y analítico para la toma de decisiones. Esta investigación puede ser utilizada como una guía para que profesores e ingenieros se den cuenta de la importancia real de las prácticas de PBL con el uso de impresoras 3D, presentadas en este estudio.

Palabras clave: Metodologías activas; Fabricación aditiva; Educación; Docencia para ingeniería; Impresión 3D.

\section{Introdução}

A metodologia ativa é um importante método para estimular os alunos a desenvolver suas habilidades, como liderança, organização e pensamento crítico. Tais habilidades são essenciais para o engenheiro ser inserido no mercado atual que exige profissionais bem preparados com práticas de gestão bem estruturadas, aliadas ao trabalho em equipe para atender suas demandas (Ponciano et al., 2017).

$\mathrm{Na}$ engenharia, o profissional lida cotidianamente com situações e projetos dos quais necessita de atitude ativa, ou seja, gerenciamento adequado com aplicações de conhecimento, ferramentas, habilidades e técnicas, além de conter um ciclo de vida com fases sequenciais cumprindo o organograma de trabalho. Assim, todo método ou estratégia que promova o envolvimento e a participação ativa do aluno de engenharia no processo de desenvolvimento do conhecimento contribui para obter ambientes ativos de aprendizagem (Campos \& Silva, 2011).

A metodologia ativa propõe o ensino por meios de projetos e por soluções de problemas, além disso essa prática favorece no aluno as atividades de ouvir, ver, perguntar, discutir, fazer e ensinar, com isso os estudantes assimilam maior volume de conteúdo, retêm a informação por mais tempo e podem aproveitar muitas aulas de maneira satisfatória (Barbosa \& Moura, 2013). Essa metodologia faz com que as informações sejam retidas por mais tempo, proporcionando um melhor aproveitamento dos estudos.

Nesse cenário de constante avanço surge a impressora 3D, ferramenta que permite a fabricação em camadas aditivas moldes de peças sólidas a partir de um design digital elaborado em diversos programas como CAD. Uma tecnologia que é empregada no desenvolvimento de projetos que surgem a partir de um modelo do objeto desejado, impresso por um aplicativo ou software em três dimensões (Santos et al., 2017). Alinhada à Engenharia é que a tecnologia é capaz de estimular as habilidades e competências de cada aluno e ajudá-lo a entender melhor o que está aprendendo, desenvolvendo seu pensamento crítico e analítico.

Nessa perspectiva, é necessário investigar o uso de Impressoras 3D com Metodologias Ativas em sala de aula, para isso foram realizadas uma entrevista estruturada com os alunos da Universidade Regional do Cariri - URCA que participaram da BRAFITEC (BRAsil France Ingénieur TEChnologie). Através das respostas obtidas foi possível validar a seguinte pergunta de pesquisa: Os participantes da BRAFITEC foram capazes de entender o uso de metodologias ativas na melhoria do processo de ensino de aprendizagem?

O presente estudo justifica-se pela necessidade atual de investir em novas técnicas de ensino e metodologias, além de possibilitar a adesão ao uso da Manufatura Aditiva (Impressão 3D) alinhada com o desenvolvimento de Metodologias Ativas para a sala de aula. Considera-se também o impacto que essas metodologias têm na aprendizagem dos alunos, bem como a capacidade de contribuir efetivamente para a prática docente do professor universitário. Nesse sentido, o trabalho tem como objetivo proporcionar novas perspectivas e trazer reflexões sobre os desafios e oportunidades para o uso desse método de ensino em Engenharia.

Assim, considera-se importante o desenvolvimento deste estudo, para que os pontos positivos e as dificuldades da 
tecnologia 3D aliadas com o uso de Metodologias Ativas para o Ensino de Engenharia possam ser discutidos. Mostrando a possibilidade de desenvolver importantes habilidades e competências nos alunos, refletindo seu impacto no ensino e na aprendizagem, permitindo assim a formação de profissionais mais críticos e analíticos para o mercado de trabalho.

\section{Metodologia}

O presente trabalho é classificado como uma pesquisa exploratória, pois tem como objetivo estudar situações em que há pouco conhecimento acumulado e, como consequência, descobrir novas práticas e melhorar novas ideias (Berto e Nakano, 2014).

A natureza deste trabalho é de caráter qualitativo. Para Yin (2016), a pesquisa qualitativa é que ela permite a realização de estudos aprofundados sobre uma ampla variedade de tópicos, incluindo seus favoritos, em termos simples e cotidianos. Além disso, a pesquisa qualitativa oferece maior liberdade na seleção de temas de interesse, porque os outros métodos de pesquisa tendem a ser limitados.

Segundo Gil (2010), os métodos e procedimentos utilizados na pesquisa são: pesquisa bibliográfica (levantamentos teóricos feitos em livros, publicações na internet) e estudo de caso (foi feito um estudo minucioso de itens de controle).

Uma leitura exploratória foi necessária para ter uma visão global do material coletado para a pesquisa. Em seguida, foi realizada a leitura seletiva, separação e análise dos materiais para agregar valor ao projeto. Os textos selecionados passaram por uma leitura analítica, com identificação e hierarquia de ideias. Também foi realizada leitura interpretativa, dando um sentido mais amplo ao objeto de estudo (Gil, 2010).

Um formulário online em formato de entrevista foi aplicado para facilitar a coleta de dados obtidos. A Entrevista foi direcionada aos alunos do curso de Engenharia de Produção Mecânica da Universidade Regional do Cariri-URCA, que tiveram contato direto com a Manufatura Aditiva, por meio do programa BRAFITEC que lhes permitiu estudar na França por um período de um ano.

Nessa experiência, os alunos aprenderam sobre o uso da impressão 3D, suas características, materiais e formas de prototipagem. Os questionários investigaram o desempenho dos alunos na experiência e sua percepção quanto ao uso dessa metodologia de ensino. Verificou-se também as dificuldades percebidas por eles e suas expectativas em relação a aulas ou oficinas, bem como as intenções de continuar utilizando a impressão 3D para projetos acadêmicos e profissionais.

Nesse cenário, podemos observar a importância de trabalhar com a manufatura aditiva no ambiente acadêmico atual. Este estudo teve como ponto de partida as seguintes questões: Como melhorar os métodos de ensino para formar profissionais com maior capacidade crítica e intervencionista? Como usar a Manufatura Aditiva para auxiliar na atualização de metodologias, permitindo maior criatividade, interatividade e interdisciplinaridade?

\section{Referencial Teórico - Metodologias Ativas}

A maioria da literatura brasileira trata as metodologias ativas como estratégias pedagógicas que colocam o foco do processo de ensino e aprendizagem no aluno, contrastando com a abordagem pedagógica do ensino tradicional centrado no professor, que transmite informações aos alunos (Valente, 2017).

Segundo Bacich e Moran (2017), metodologias ativas são estratégias de ensino centradas na participação efetiva dos estudantes na construção do processo de aprendizagem, de forma flexível, interligada e híbrida. A compreensão, a escolha e o interesse são condições essenciais para ampliar as possibilidades dos estudantes de exercitar a liberdade e a autonomia na tomada de decisões em diferentes momentos do processo que vivência, preparando-se para o exercício profissional futuro (Faria, 2021). 
Os cursos de graduação vêm buscando concepções e metodologias de ensino que possibilitem práticas pedagógicas orientadas pela concepção do estudante como sujeito ativo, nessa procura é citado o PBL (Freitas, 2012).

Os cursos de Ensino Superior, vêm buscando concepções e metodologias de ensino que possibilitem práticas pedagógicas voltadas para o estudante como sujeito ativo, este por sua vez tem uma tarefa que ele somente poderá realizar se mobilizar conteúdos para efetuar a aprendizagem de uma maneira ativa e autônoma. As situações problema podem engendrar uma grande variedade de práticas pedagógicas no ensino superior; podem, ainda, associar-se a outra das metodologias ativas de ensino: a chamada aprendizagem baseada em problemas, PBL, do inglês, "Problem-Based-Learning" (Diniz, 2018).

Desenvolver abordagens novas para acompanhar novos tempos é extremamente importante e necessário para aprimorar a educação, pois não se pode negar a quantidade assombrosa de modificações sociais que presenciamos nos últimos anos. Alguns termos são comuns à ideia das metodologias ativas: a aprendizagem ativa se refere ao engajamento dos estudantes com as tarefas de aprendizagem, de forma a continuamente refletir sobre o que está sendo aprendido, questionar os resultados alcançados e os seus próprios caminhos de aprendizagem. Outro termo, a aprendizagem centrada no estudante, entende que esse deve poder influenciar/opinar do conteúdo a ser aprendido aos métodos de avaliação, equilibrando a relação de poder sobre o que será aprendido e como, com o professor (Júnior et al., 2019).

De modo geral, toda a aprendizagem é ativa em um determinado grau, porque exige do educando e do educador formas diferentes de movimentação interna e externa. "A aprendizagem mais profunda requer espaços de prática frequentes e de ambientes ricos em oportunidades" (Moran, 2018, p. 3).

As metodologias ativas têm sido aplicadas em vários contextos, como uma maneira de lidar com as dificuldades, principalmente em disciplinas das áreas de exatas, Engenharia e físicas, e ambiguidades que inevitavelmente podem existir, quando os problemas de ensino-aprendizagem são abordados sob uma perspectiva do desenvolvimento cognitivo (Garmendia et al., 2015; Varela et al., 2017; Forndran \& Zacharias, 2019).

É necessário que haja mais ações efetivas para implementação de metodologias ativas e se possível diversificadas nos cursos de engenharias. O mercado está cada vez mais exigente, já que as tecnologias estão evoluindo e os profissionais precisam acompanhá-las com as suas competências e habilidades adquiridas no decorrer do curso (Miranda \& Bortoluzzi, 2018).

\subsection{Manufatura Aditiva}

O termo Indústria 4.0 foi criado e disseminado pelos alemães no ano de 2011, durante uma importante feira de tecnologia, que contou com o apoio do governo e de indústrias do próprio setor. Esse termo faz parte de um conjunto de estratégias que desafiam as indústrias a realizarem uma espécie de "4" Revolução Industrial", de modo que todo o seu processo seria interconectado por meio de redes inteligentes, tornando a mais eficaz e competitiva, e em um segundo momento, permitindo que esses processos produtivos sejam capazes de governarem a si mesmos (Prado et al, 2019).

Nos dias atuais as empresas buscam investir cada vez mais na questão da inovação e em novos mecanismos, capazes de favorecer o desenvolvimento e elevar o padrão das organizações. Estas por sua vez, visam atingir destaque no mercado competitivo com processos de produção cada vez mais eficientes, favorecidos pela era tecnológica (Campos \& Silva, 2011).

A Manufatura Aditiva, também conhecida como impressão 3D, é uma tecnologia que permite a fabricação de peças com formas complexas, através do auxílio de software e hardware característico para a produção. Em outras palavras, o processo de fabricação consiste em projetar e fabricar o modelo de um produto ou peça a ser construído, podendo criar formas geométricas complexas em um curto espaço de tempo de fabricação (Hilário, 2016).

$\mathrm{O}$ uso das tecnologias da manufatura aditiva (MA) se encontra em alta nos diversos campos do mercado industrial. Proveniente de um processo de impressão de objetos visivelmente palpáveis, a manufatura aditiva é utilizada em diversas áreas 
e atualmente tem destaque nos setores automotivos e aeroespacial, e também nos projetos de implantes odontológicos (Marques, 2014).

De acordo com Baygin et al., (2016) os termos Fábrica Inteligente, Fábrica do futuro, Fábrica Inteligente usada na indústria 4.0 contabilizam como as fábricas serão no futuro, mais dinâmicas e inteligentes, mais ágeis e flexíveis, fabricando produtos inteligentes, utilizando equipamentos inteligentes englobados em processos inteligentes de produção (Huba \& Kozák, 2016).

Dos Santos et al. (2019) afirmam que o avanço tecnológico ocorre em vários seguimentos da sociedade, com o uso de novas tecnologias em diversos setores. A tecnologia de impressão 3D vem crescendo rapidamente mudando o processo de e estrutura de inovação, sendo sua utilização uma consequência de avanços tecnológicos mundial e do surgimento de novas tecnologias (Woodson et al., 2019).

\subsection{Prototipagem na Engenharia}

De acordo com Baygin (2016), A Rapid Prototyping é uma tecnologia que possibilita a produção de modelos e protótipos diretamente do modelo 3D (obtido por um sistema CAD, por meio de digitalização ou por Tomografia Computadorizada).

A palavra protótipo é derivada do prototypon grego cujo significado é a primeira forma (Grimm, 2004) ou o primeiro produto produzido (Floyd, 1984). Esta definição considera que um protótipo implica a pré-produção completa do produto.

De acordo com Lee (1998) o modelo protótipo de um novo produto pode ser feito a partir do modelo CAD desenvolvido em Engenharia Reversa usando a técnica de Prototipagem Rápida.

Genericamente, em Rapid Prototyping (Prototipagem Rápida), os modelos físicos são fabricados camada por camada. Este sistema aceita um arquivo de malha triangular no formato STL. Este formato tem vantagens devido à sua estrutura simples e fácil de usar, mas tem sérias desvantagens. Ele precisa de um alto índice de memória dependendo da precisão do modelo. Às vezes, muito tempo é gasto com reparos, quando o modelo apresenta lacunas, sobreposições e vetores com problemas (Jorge et al., 2019).

A impressora 3D vem como uma ferramenta de suporte que elimina os custos de retrabalho dos moldes de madeira ou alumínio através da impressão com material ABS (Acrilonitrile Butadiene Styrene), um método que permite uma prévia do design final sem o investimento do molde por terceiros (Dantas et al., 2018).

Nas etapas de design e detalhamento de produtos e engenharia de processos, os protótipos iniciais são tipicamente produzidos a partir de esboços, desenhos de produtos 3D e o uso de prototipagem rápida (Saffaro, 2007).

Como parte desse processo, a prototipagem é relevante porque visa proporcionar aos alunos diferentes perspectivas sobre o processo de projeção e maturação de soluções para que sejam relevantes no contexto analisado. Após a criação de protótipos que representam as melhores soluções criadas, os grupos saem novamente em campo para ouvir diferentes perspectivas sobre os protótipos (Oliveira et al., 2017).

\subsection{Ensino por meio de Impressoras 3D}

Os primeiros estudos com impressora 3D datam de 1970, iniciando a indústria de impressão no final dos anos 80, na época em que as impressoras tinham alto custo. A diferença entre o novo método utilizado, para o que existia na época, é o fato de que eles imprimiram camadas por camadas, tornando-se uma produção aditiva, o método que existia era subtrativo, ou seja, retirava o material dando forma ao modelo final (Kietzmann et al., 2015).

Impressoras 3D permitem prototipagem rápida de objetos personalizáveis. Com a popularização da tecnologia hoje, essa característica favorece os professores a produzirem seus próprios materiais educativos, atendendo de forma mais assertiva 
as demandas que observam em suas práticas em sala de aula (Onisaki \& Vieira, 2019).

Durante as atividades realizadas com a tecnologia, é possível perceber o grande entusiasmo e concentração dos alunos diante das demonstrações utilizando a impressora 3D e, além disso, os benefícios para a melhoria da aprendizagem são visíveis e viáveis (Onisaki \& Vieira, 2019).

A interação com outras pessoas que estão desenvolvendo projetos, de diversos níveis educacionais e diferentes origens, como estudantes do ensino médio, universitários, engenheiros, arquitetos e artesãos, colabora expressivamente para otimizar o processo de aprendizagem, pois compartilham suas experiências (Onisaki \& Vieira, 2019).

A "Impressora 3D" é uma tecnologia tão atual quanto à necessidade de metodologias ativas no ensino e pode abranger a aplicação do conhecimento teórico da sala de aula, bem como desenvolver habilidades transversais. Além disso, possibilita a parceria entre diferentes ramos e cursos da universidade, um contato necessário, pois reflete a interdependência entre áreas distintas do conhecimento (Bedeschi et al., 2017).

Bedeschi et al., (2017), também afirmam que o ensino pode ser melhorado por meio de atividades práticas, especialmente quando conceitos difíceis de visualizar e abstrato estão envolvidos, mesmo com a ajuda de simulações virtuais em computadores.

Os modelos 3D impressos ampliam o campo de mediação de elementos no processo de aprendizagem, devido à sua empregabilidade como instrumento didático. "Saber usar essa tecnologia no ensino possibilita transformar impressoras 3D em pequenas fábricas de ferramentas de ensino para serem utilizadas em atividades práticas" (Aguiar, 2016, p. 24), o que certamente facilita o trabalho docente e desmistifica sua prática maximizando o campo de atuação do aluno.

O uso do recurso impresso em 3D juntamente com a teoria, pode facilitar a reflexão e o aprofundamento do conteúdo teórico, pois a visualização do mesmo permite ao aluno manipular o material, visualizando-o de vários ângulos, melhorando assim sua compreensão do conteúdo abordado (Orlando et al., 2009).

Assim, percebe-se que os recursos impressos em 3D são materiais didático-pedagógicos alternativos para facilitar a compreensão do ensino, associado às aulas teórico-práticas proporciona melhor compreensão ao aluno, por meio da observação, análise e manipulação desses materiais, uma vez que a aprendizagem é mais eficaz quando confrontada com o material do estudo (Orlando et al., 2009)

Assim, formal ou informalmente, por meio da institucionalização metodológica do ensino ou pela iniciativa privada de professores, há uma série de ações e propostas de trabalhos que buscam inter-relacionar conteúdos didaticamente dispersos e fragmentados, mas fortemente imbricados na realidade (Braida, 2014, p.143).

\section{Resultados e Discussão}

Com a aplicação do formulário com os nove alunos da URCA que participaram do programa BRAFITEC (Brasil, França), foi possível obter alguns dados sobre a importância dessa ferramenta no ensino e aprendizagem. O formulário continha perguntas abertas e fechadas para identificar, analisar e entender melhor os pontos fortes e encontrar dificuldades no uso da impressão 3D em sala de aula.

Os resultados do Formulário Online foram monitorados em relação à execução das atividades e ao processo de ensino e aprendizagem dos alunos entrevistados. Os resultados das questões 1, 2, 3 e 4 são identificados abaixo:

\section{Item 1 - Avaliação dos alunos em relação aos impactos da ferramenta como instrumentação do ensino}

Neste item os alunos descreveram pontos relevantes como por exemplo a materialização das ideias desenvolvidas e o uso da integralização (teoria x prática) dos conhecimentos adquiridos; a junção da praticidade e rapidez de elaboração de peças mais complexas, definindo o método de Impressão 3D em suas respostas como eficaz no que diz respeito ao alcance de tirar os 
projetos do papel e conseguir construir diversos protótipos. Pontuaram também observações feitas sobre a idealização na construção de um produto e o uso constante do FabLab para produzir os protótipos. Para eles, estas práticas tornam possível o incentivo contínuo de todos, passando uma sensação de real domínio na entrega dos projetos, além da facilidade de ensino e aprendizagem para os estudantes.

A percepção da criatividade e a prototipagem rápida no âmbito de projetos inovadores, seguida da flexibilidade de criação para aumentar a possibilidade de projetar estudos em andamento, foram colocados também como fatores determinantes para a construção dos conhecimentos dos alunos durante a vivência das aulas/oficinas.

\section{Item 2 - Metodologias ativas com o uso da Manufatura Aditiva e o aprimoramento das habilidades e competências dos alunos}

Neste item foi verificado que $100 \%$ dos alunos entrevistados, concordaram plenamente com o impacto positivo das metodologias ativas acompanhadas pela Impressão 3D para melhorar as habilidades e competências desenvolvidas pelos estudantes envolvidos.

Isso demonstra perfeitamente a total eficiência da metodologia de ensino para a melhoria contínua da aprendizagem dos alunos, os conhecimentos empregados em sala de aula, pois é essencial trabalhar com o desenvolvimento de suas habilidades e competências, garantindo cada vez mais a formação de profissionais conscientes e mais autônomos diante da resolução dos problemas que existem no dia-a-dia, enquanto profissional.

Verificou-se também que para produzir os objetos é utilizado apenas o material necessário, salvando a matéria-prima, ou seja, há o combate ao desperdício. Além disso, o custo da mão-de-obra é reduzido e os profissionais podem ser utilizados em tarefas mais estratégicas dentro das empresas, valorizando assim o uso de habilidades e competências já aprimoradas.

Outro ponto importante é a possibilidade de inovar, pois o uso de Impressoras 3D, permite a personalização de peças e em um cenário de negócios, onde se é cada vez mais competitivo, inovar é uma questão de sobrevivência. Portanto, as barreiras para criar novos negócios e produtos tornam-se invisíveis quando você tem em suas mãos uma máquina que pode criar (quase) tudo. Assim, sua aplicação na educação proporciona a absorção de conteúdos mais complexos, tornando a sala de aula em um ambiente mais interativo e didático.

\section{Item 3 - Avaliação do ambiente onde as atividades foram desenvolvidas e os projetos em Impressão 3D concebidos}

No Item 3 nota-se que $100 \%$ dos alunos que responderam ao formulário estão satisfeitos e de acordo com o ambiente onde as atividades e projetos foram desenvolvidos. Neste item observa-se a importância de se ter um ambiente adequado e propício a realização das atividades, tendo em vista o propósito de fazer o melhor pelos estudantes, contando com alta tecnologia e equipamentos modernos, para então acompanhar a evolução das técnicas, utilizando materiais de extremo controle de qualidade.

Os alunos descreveram um ambiente bem equipado com mesas, cadeiras e projetores, onde todo o espaço era pensado para motivar a criatividade e inovação, com uma variedade de impressoras 3D de distintas especificações, mais uma vasta exposição de protótipos e projetos realizados anteriormente por equipes de pesquisa e com suporte técnico sempre disponível por especialistas do próprio laboratório. Citaram que as atividades eram desenvolvidas em um laboratório (FabLab) próprio para inovação e prototipagem e que além de impressoras 3D, haviam também o corte laser, scanner 3D, máquinas CNC, salas próprias para fazer brainstormings, além de extrusoras para reciclagem de plástico, ou seja ambiente que propicia a maior interação entre as pessoas, voltado para o desenvolvimento e discussão de ideias, com suporte e disposição do material necessário, professores bem qualificados e de uma equipe técnica bem capacitada. 


\section{Item 4 - Criação e testagem de protótipos físicos no ambiente onde as atividades eram realizadas}

Ao analisar o Item 4, foi possível observar como os alunos poderiam desenvolver e intensificar seus projetos, considerando a apropriação do conhecimento e sua própria autonomia na execução desses. Observa-se que $100 \%$ dos alunos tinham total liberdade para criar os protótipos físicos, de acordo com as habilidades exigidas em cada atividade.

Os alunos especificaram como acontecia a criação de seus protótipos, descrevendo pontos importantes para a construção deles. Eles relatam que utilizavam a impressão em pó, PLA, ABS, SLA e ABS reciclado em local, sob o auxílio teórico (sala de aula), além do desenvolvimento em CAD e materialização (Impressão 3D), tudo isso sob a supervisão dos membros do Fab Lab e professores da IES. Caracterizaram as atividades com a ferramenta como uma experiência de máxima agregação de valor (para todas as partes envolvidas), enriquecedora já que a maioria desses projetos eram realizados em grupos, onde era possível a discordância dos colegas e propor suas próprias ideias, enfim uma ótima oportunidade para analisar o projeto sob diferentes perspectivas e olhares.

Foi realizado ainda estudos dos materiais e definida a estrutura física do tecido para que se garantisse maleabilidade e elasticidade, definido o uso dos protótipos de um tecido feito inteiramente em Impressora 3D. Foram feitos testes mecânicos, usavam-se faces escaneadas, chaveiros e pequenos objetos em scanner. Os alunos do BRAFITEC entendem que a impressão $3 \mathrm{D}$ veio para oferecer uma alternativa aos diversos tipos de processos e principalmente: reduzir custos. Empresas de diversas áreas de atuação têm usado tal método, resultando em modelos mais baratos e pondo fim ao desperdício de matéria-prima.

São apresentados os seguintes resultados referentes à execução das atividades, implantação da ferramenta no curso de Engenharia da URCA e incentivo à criatividade dos alunos entrevistados. Os resultados das questões 5,6 e 7 estão descritos nos respectivos itens:

\section{Item 5 - Incentivo à criação dos próprios projetos desde a concepção até o protótipo final}

No item acima pode- se observar o potencial da ferramenta, considerando o incentivo da criatividade, em relação a garantir a criação de projetos únicos, exclusivos e mais estruturados. Percebe-se que a maioria dos estudantes são incentivados a criarem seus próprios projetos desde a concepção até o protótipo final, o que torna possível o desenvolvimento de uma aprendizagem estruturada frente a Manufatura Aditiva. Os estudantes relataram também que durante as aulas, são desenvolvidas habilidades como criatividade, interesse, organização, além do desenvolvimento da sua capacidade crítica e analítica, o que remete a todos, encontrarem soluções para os dilemas presentes no cotidiano relacionando teoria e prática, validando de fato a eficiência desta metodologia como ferramenta para construção do conhecimento.

Com base nas respostas dadas, verificou-se as principais características da impressão 3D que motivam o seu uso. Os alunos apontaram características relacionadas a praticidade para replicar e criar projetos, pontuaram também a rapidez de impressão e o fato de poder trazer objetos reais para a realidade, além da complexidade das peças produzidas, agregação de valor a peças desgastadas e, em níveis gerais, perspectivas relacionadas a sustentabilidade.

Alguns dos estudantes descreveram o material como de fácil acesso, observando a possibilidade de visualizar projetos reais em protótipos e com isso trabalhar modificações, conseguindo realizar qualquer tipo de ajuste de acordo com os pontos importantes para a impressão. Falaram ainda sobre a alta flexibilidade e o baixo custo de prototipagem, dando ênfase ao processo de prototipagem rápida, alta personalização e riqueza de detalhes.

\section{Item 6 - Possibilidade de usar a criatividade dos estudantes alinhada as técnicas de Impressão 3D}

No Item 6 os alunos do BRAFITEC, descreveram a questão da criatividade em torno das técnicas de Impressão 3D, na garantia da fabricação de projetos únicos, exclusivos e bem estruturados. Percebe-se também que a maioria dos alunos avaliaram positivamente a Manufatura Aditiva, no que compete o desempenho de suas atividades na sistematização de 
soluções para os problemas apresentados, considerando maior liberdade para produzir objetos complexos ou ideias mais "extravagantes", pois tal ferramenta tornou evidente a certeza de que no fim seria possível a materialização das ideias.

Os estudantes relatam que as atividades com a Manufatura Aditiva eram exploradas a partir de brainstorming's e questionamentos quanto ao design (projeto) do produto e as suas complexidades dimensionais/ funcionais, eles relatam que eram desafiados a criar a partir da exposição de ideias e competições internas vários tipos de protótipos, isto desde a criação de objetos lúdicos a modelos úteis aplicados na Medicina e Engenharia. Dando destaque também sobre a questão do laboratório, que era utilizado exclusivamente para as atividades, mostrando a real importância da existência de um espaço propício e adequado para o desenvolvimento das práticas envolvendo a Impressão 3 D.

Comparado aos processos tradicionais a prototipagem 3D torna o processo mais dinâmico, possibilitando mais interações e modificações, além de ser mais barato para pequenas quantidades. Fica claro que a impressão 3D vem então como um formato alternativo para o processo de idealização e prototipagem de um produto, tornando possível a sua utilidade como ferramenta para o processo de ensino e aprendizagem dos estudantes de Engenharia.

\section{Item 7 - Utilização de Impressão 3D nas disciplinas do curso de Engenharia de Produção Mecânica da URCA}

O item 7 trata do percentual de aceitação dos alunos a respeito da implementação de atividades que incluem o uso da Impressão 3D, nas disciplinas do curso de Engenharia de Produção Mecânica da URCA (Universidade Regional do Cariri). Neste quesito há uma excelente aceitação dos alunos sobre a inserção da ferramenta no curso de Engenharia da Universidade.

Os alunos mencionam os principais benefícios da Manufatura Aditiva para o curso e disciplinas, bem como os aspectos impactantes na aprendizagem, isso observando a natureza do curso e como indispensável a produção de protótipos nas disciplinas da Matriz Curricular de Engenharia de Produção Mecânica se tornaria indispensável. Eles acreditam firmemente que a impressão 3D pode melhorar a capacidade de aprendizagem dos alunos, a partir de suas experiências através da BRAFITEC, mostrando assim a relação entre (teoria x prática), o que permitiria aos graduandos preparar novas demandas de fabricação/indústria 4.0, já que essa tecnologia é considerada emergente e já está presente em diversas empresas.

Os alunos também destacam o currículo focado em pesquisa e desenvolvimento de projetos no MA, aumentando o número de artigos científicos na área, auxiliando em aulas envolvendo as disciplinas de mecânica do curso e criando peças para representar uma variedade de projetos e produtos. A Manufatura Aditiva para eles seria útil não só para o curso de Engenharia, mas também para todos os alunos que quisessem desenvolver outros tipos de projetos em outras áreas especificas de outros cursos. Uma ferramenta como essa agregaria valor ao curso não só para Impressão 3D, mas também pelo fato de ter uma forte visibilidade em torno da instituição por ter um laboratório FAB adequado e bem equipado para realizar as atividades.

Por fim, apresentamos os resultados relacionados ao conhecimento adquirido nas aulas práticas, os mais utilizados durante o processo de prototipagem e as técnicas de prototipagem mais utilizadas durante o programa. Os resultados das questões 8,9 e 10 estão representados nos seguintes itens:

\section{Item 8 - Conhecimento adquirido e vivenciado durante aulas e oficinas com o uso de Impressoras 3D}

Neste item nota-se excelente aceitação dos alunos, quanto ao uso da ferramenta como suporte para a construção do conhecimento adquirido em sala de aula. Destaca-se também a partir dos comentários feitos a importância da Manufatura Aditiva para a estruturação e apropriação do conhecimento adquirido, considerando que a maioria dos alunos demonstraram grande satisfação em desenvolver seus projetos e resolver as questões presentes nos problemas apresentados.

Considerando as respostas dos alunos da BRAFITEC, podemos compreender plenamente o papel das Metodologias Ativas em sala de aula e como o conhecimento tem sido disseminado para eles em seu contato direto com a metodologia. Os alunos pontuaram que as ideias, em níveis teóricos, foram desenvolvidas em uma sala sob a participação dinâmica dos 
envolvidos e, em níveis práticos, sua materialização foi sob orientação e apoio da equipe técnica do laboratório (especializada em impressão 3D) da instituição. Também compartilharam que na fase inicial foram ministradas aulas teóricas, com exposição de algumas técnicas e estudos de caso bem aplicados, seguidas posteriormente por aulas práticas em laboratório, com a proposição de projetos práticos de estudos com maior frequência em grupos, onde deveriam criar uma solução para um determinado problema ou a criação de um novo produto.

O laboratório da instituição de Impressão 3D (Fab Lab), recebeu os modelos via pen drive e assim os profissionais do laboratório os ajudaram a imprimir os protótipos. Eles detalham que após a obtenção do modelo 3D em formato Stl, foi necessário passar os modelos através do software fatiador 3D e escolher os parâmetros necessários para impressão (tamanho da peça, \% de enchimento, velocidade, temperatura de extrusão, temperatura da câmara, suporte ou não, primeira camada, formato de preenchimento interno, quantidade de camadas externas, etc...). Após esta etapa, a impressora foi verificada para atingir a temperatura ideal, uma vez que a temperatura foi atingida, a impressão então é liberada.

\section{Item 9 - Materiais mais utilizados no processo de prototipagem com Impressão 3D, em aulas e oficinas BRAFITEC}

$\mathrm{O}$ item 9 mostra os materiais mais utilizados em experiências com Impressão 3D, vivenciadas pelos alunos da BRAFITEC. É muito importante buscar compreender as principais características de cada tipo de material e, com base neles, selecionar o melhor material para que sua impressão seja de qualidade e cumpra com seu objetivo.

Entre os materiais listados para a fabricação de protótipos no laboratório do programa, os alunos indicaram como mais utilizados: PLA Plastic (83\%), ABS Plastic (66,7\%), Resina (53\%) e PET Plastic (33,3\%).

Algumas considerações devem ser levadas em consideração de acordo com a literatura e as experiências obtidas pelos alunos:

- Se o aluno é iniciante na área de Manufatura Aditiva, o PLA é o material recomendado para suas primeiras impressões, pois permite que ele adquira experiência e identifique onde melhorar, sem comprometer de forma muito significativa o resultado da peça.

- O uso do polímero ABS, mostrou-se extremamente eficiente quando aplicado à Impressão 3D, uma vez que possui boa resistência mecânica e térmica, além de permitir o acabamento após a fabricação, desde lixamento ou banho de acetona.

- A resina de impressão 3D padrão ou standard é um material que oferece ótima qualidade a um preço baixo, tornando-se uma excelente opção de custo benefício. A resina padrão também tem um alto nível de detalhes, é translúcida, suave ao toque e fácil de pintar e finalizar.

- PET é um material considerado ideal para o processo de Manufatura Aditiva, considerado o substituto do ABS em um futuro de curto a médio prazo. É um termoplástico adequado para Impressões 3D que requerem resistência ao impacto e robustez da peça. Também possui excelente resistência térmica, exigindo temperaturas de extrusão em torno de $240{ }^{\circ} \mathrm{C}$.

Como já mencionado neste estudo, a Manufatura Aditiva também está a serviço da sociedade, pois carrega em sua essência a alta capacidade de inovação e adaptação do processo às necessidades das pessoas em diversos setores da sociedade. Portanto, é notável, que diferentes aplicações requerem diferentes materiais e cabe ao usuário essa decisão e conhecimento, com o objetivo de entregar a mais alta qualidade possível vinculada ao seu projeto.

\section{Item 10 - Técnicas de prototipagem que os alunos já conheciam antes de participar do programa BRAFITEC e projetos com Fabricação Aditiva}

O item 10 verificou o conhecimento prévio dos alunos sobre as técnicas de prototipagem utilizadas no programa. Os alunos indicaram as seguintes opções para impressão 3D e corte a laser: Ambos os métodos (50\%), Impressão 3D (16,7\%), Apenas corte a laser $(16,7 \%)$ e Nenhum dos dois $(16,6 \%)$. 
A prototipagem tem sido uma das atividades mais utilizadas pelas indústrias quando se trata de impressão 3D, o que mostra a importância do uso de técnicas de prototipagem para acelerar o desenvolvimento de produtos e reduzir os custos de lançamento de novos produtos no mercado.

Quanto a prototipagem rápida, os alunos descreveram que em níveis experimentais, eles usavam para criar modelos finais a Impressão 3D do início ao fim, e nas aulas: para a criação de blocos de teste, peças para criar dispositivos para melhor adaptar um sistema de medição para forças de perfuração, bem como a criação de simuladores cirúrgicos, objetos recreativos na engenharia, e brinquedos. Em alguns casos, a impressão foi utilizada na fase final do projeto, eles detalham a experiência com essa tecnologia como fantástica, pois era algo novo, inovador e interessante, pois muitos não estavam acostumados a essa forma de trabalho, descrevem a experiência como algo tão gratificante no sentido de suas próprias ideias poderem ter sido materializadas.

Percebeu-se que a impressão 3D estava presente em todas as etapas dos projetos. Os alunos descrevem e classificam a Manufatura Aditiva como uma grande oportunidade para inovar e aprender cada vez mais, pois a ferramenta permite ensaiar diferentes perspectivas a baixo custo, facilitando a realização da necessidade de implementação de ajustes ao longo dos projetos.

\section{Considerações Finais}

O estudo apresentado surgiu a partir da problemática envolvendo o uso de Impressoras 3D para o desenvolvimento de Metodologias Ativas em cursos de Engenharia, por meio de um contexto em que o desenvolvimento econômico demanda cada vez mais profissionais de engenharia que possuam habilidades e competências que atendam as diversas exigências do mercado de trabalho atual.

Houve também a necessidade de conhecer e mostrar a importância da Manufatura Aditiva combinada com as Metodologias Ativas, como método benéfico para a construção da aprendizagem e domínio de novas tecnologias, garantindo assim a formação de profissionais mais críticos e reflexivos, dotados de habilidades sociais, pessoais, metodológicas e de determinadas técnicas para resolução de problemas.

Neste artigo, um estudo foi desenvolvido por meio de pesquisa bibliográfica realizada nas principais bases de dados e por meio de uma entrevista aplicada, com os alunos do programa CAPES - BRAFITEC, que promove o intercâmbio de alunos em todas as especialidades de engenharia, garantindo que eles estudem até um ano de sua graduação na França. Assim, foi possível responder e validar a questão da pesquisa, pontuando como o uso de Metodologias Ativas, alinhadas com a Manufatura Aditiva conecta e possibilita que os projetos desenvolvidos pelos alunos, garantam aulas interativas e dinâmicas, fortalecendo o ensino e a aprendizagem em sala de aula, onde os mesmos são incentivados a desenvolver pensamentos críticos e analíticos para a tomada de decisão.

A partir desta pesquisa pode-se ver que a Impressão 3D inserida como ferramenta metodológica tem potencial favorável para maximizar a aprendizagem dos alunos, tornando a sala de aula um ambiente mais atraente e dinâmico, além de tornar a aprendizagem mais eficaz, dando aos alunos total liberdade para criar e desenvolver seus próprios protótipos, considerando a criatividade e imaginação. Percebe-se também através das tarefas desenvolvidas, como o uso da modelagem e a visualização de peças impressas, que os alunos do BRAFITEC, puderam vivenciar experiências através de um contato direto com o exercício da profissão, o que de fato os estimula e permite que eles se diferenciem do padrão de formação, pois não adquirem apenas o conhecimento teórico à medida que o aplicam para a solução de problemas e à implementação de inovações.

A "Impressora 3D" é uma tecnologia tão atual quanto a busca de Metodologias Ativas no ensino e pode abranger a aplicação do conhecimento teórico da sala de aula de forma prática, bem como desenvolver habilidades transversais. Além 
disso, possibilita a parceria entre diferentes ramos e cursos da universidade, um contato necessário, pois reflete claramente a interdependência entre as diferentes áreas do conhecimento.

Espera-se que esta pesquisa tenha impacto e contribua para a comunidade acadêmica e científica, garantindo o desenvolvimento de novas pesquisas na área. Como sugestão para futuras pesquisas, sugere-se a implementação para o estudo da aplicação da ferramenta em disciplinas de Engenharia de forma específica e individualizada, bem como análises adicionais sobre o impacto dessa metodologia de ensino no desempenho acadêmico dos alunos de graduação.

Outra sugestão para trabalhos futuros seria um estudo com a Manufatura Aditiva, envolvendo professores e alunos, a partir da interação com o que é feito na universidade, juntamente com as necessidades do mercado ou as necessidades da comunidade, fortalecendo ainda mais o papel da instituição, garantindo incentivo tecnológico e inovação.

\section{Referências}

Aguiar, L. D. C. D. (2016). Um Processo Para Utilizar A Tecnologia De Impressão 3d Na Construção De Instrumentos didáticos para o ensino de ciências. Journal of Chemical Information and Modeling, 6 (2), 1689-1699. http://hdl.handle.net/11449/137894

Bacich, L., \& Moran, J. (2017). Metodologias ativas para uma educação inovadora: uma abordagem teórico-prática. Penso.

Baygin, M., Yetis, H., Karakose, M., \& Akin, E. (2016). An effect analysis of industry 4.0 to higher education, no ITHET: Information. Technology. Based Higher. Education. and Training, 15th International Conference on. IEEE. 1-4. 10.1109/ITHET.2016.7760744

Barbosa, E. F., \& Moura, D. G. (2013). Metodologias Ativas de Aprendizagem Na Educação Profissional e Tecnológica. Boletim Técnico Senac, 39 (2), 48 67. https://doi.org/10.26849/bts.v39i2.349

Berto, R. M. V. S., \& Nakano, D. (2014). Revisitando a produção científica nos anais do Encontro Nacional de Engenharia de Produção. Production, 24, 225232. https://doi.org/10.1590/S0103-65132013005000007

Borges, T. S., \& Alencar, G. (2012). Metodologias ativas na promoção da formação crítica do estudante: o uso das metodologias ativas como recurso didático na formação crítica do estudante do ensino superior. Cairu em Revista, 3 (4), 119-143.

Bedeschi, A. C., et al. (2017). Contribuições Do Desenvolvimento De Sistemas De Impressão 3D Na Educação. COBENGE: XLV CONGRESSO Brasileiro De Educação Em Engenharia, Joinville/SC, 1-10.

Braida, F. (2014). Da Aprendizagem Baseada em Problemas à Aprendizagem Baseada em Projetos: estratégias metodológicas para o ensino de projeto nos cursos de Design", In: Actas de Diseño. Diseño en Palermo, 17 (17), 142-146. https://doi.org/https://doi.org/10.18682/add.vi17

Campos, L., \& Silva, J. (2011). Aprendizagem Baseada em Projetos: uma nova abordagem para a Educação em Engenharia. COBENGE 2011: XL Congresso Brasileiro de Educação zem Engenharia, Santa Catarina, 1-4.

Dantas, I. M., et al. (2018). Implantação De Impressão 3D: Melhoria No Processo De Projetos No Grupo Açotubo. Revista Científica Semana Acadêmica, 3, 1-17. https://semanaacademica.org.br/artigo/implantacao-de-impressao-3d-melhoria-no-processo-de-projetos-no-grupo-acotubo

Diniz, J. F. (2018). Metodologias ativas no ensino superior: a articulação da resolução de situações problema com o ensino por meio de projetos em prática. revista ensaios pioneiros, $32-46$

Forndran, F., \& Zacharias, C. R. (2019). Gamified experimental physics classes: a promising active learning methodology for higher education. European Journal of Physics, 40 (4). http://dx.doi.org/10.1088/1361-6404/ab215e

Faria, G. A. D. S. (2021). O ensino de química por meio de metodologias ativas no contexto dos polímeros e agrotóxicos. Dissertação de Mestrado em Universidade Federal de Minas Gerais para Mestrado em Educação, Faculdade de Educação, Mestrado Profissional em Educação.

Floyd, C. (1984). A systematic look at prototyping. In: BUDDE, R. (Ed.); Kuhlenkamp, K. (Ed.); Zulligohoven, H. (Ed.), Approaches to prototyping. Berlin: Springer Verlag, 2, 12-20.

Freitas, R. (2012). Ensino por problemas: uma abordagem para o desenvolvimento do aluno. Educação e Pesquisa, São Paulo, 38 (2), $403-418$. https://doi.org/10.1590/S1517-97022011005000011

Garmendia, M., Aginako, A. Z., \& Solaberrieta, M. E. (2015). Engineering students perceptions about active teaching methodologies in the university of the basque country. Iceri2015: 8th International Conference of Education, Research and Innovation, Sevilha, 7922-7931. https://library.iated.org/publications/ICERI2015/start/225

Gil, A. C. (2010). Como elaborar projetos de pesquisa. Atlas.

Grimm, T. (2004). User's guide to rapid prototyping. Dearborn, Society of Manufacturing Engineers, Miami.

Hilário, L. T. G. (2016). Desenvolvimento de processo de fabricação de compósitos de fibras longas através da tecnologia de Manufatura Aditiva, Tese de Doutorado em Engenharia Mecânica, Universidade de São Paulo - USP, https://doi.org/10.11606/T.18.2019.tde-04092019-125437 
Huba, M., \& Kozák, Š. (2016). From. E-learning. to Industry. 4.0. In: Emerging eLearning Technologies and Applications. ICETA: International Conference on. IEEE. 103-108. Doi: 10.1109/ICETA.2016.7802083

Yin, R., (2016). Pesquisa Qualitativa do Início ao Fim. Penso.

Jorge, J. M., Firmino, A. T., \& Santos, A. C. (2019). Análise Da Impressão 3D No Ensino De Engenharia: Um Estudo De Caso No Laboratório Aberto De Brasília. CBGDP: $12^{\circ}$ Congresso Brasileiro de Inovação e Gestão de Desenvolvimento do Produto, Unb, Brasília, 2 (6), 946-956. 10.5151/cbgdp2019-69

Junior, J. M. A., Souza, L. P., \& Silva, N. L. C. (2019). Metodologias ativas: Práticas pedagógicas na contemporaneidade. Editora Inovar.

Kietzmann, J., Pitt, L., \& Berthon, P. (2015). Disruptions decisions and destinations: Enter. the age. of 3D printing and additive. Manufacturing. Business Horizons, 58 (2), 209-215. 10.1016/j.bushor.2014.11.005

Lee, K.H., \& Woo, H. (1998). Use of Reverse Engineering Method for Rapid Product Development. Computer Computers \& Industrial Engineering. Elsevier Science Ltd, 35, 21-24.

Marques, K. (2014). Manufatura aditiva: o futuro do mercado industrial de fabricação e inovação, Tese de Doutorado em Engenharia Mecânica, EESCEscola de Engenharia de São Carlos, USP-Universidade de São Paulo.

Miranda, M. R. S., \& Bortoluzzi, M. B. O. (2018). Perspectivas Em Diálogo Revista de Educação e Sociedade Perspectivas. Revista Educação e Sociedade, 5 (9), 282-283.

Moran, J. (2018) Metodologias ativas para uma aprendizagem mais profunda. In: Bacich, L.; Moran, J. Metodologias ativas para uma educação inovadora: uma abordagem teórico-prática. Penso.

Oliveira, C. M. DE et al. (2017). A importância das atividades práticas no ensino de Engenharia do Produto: Um exemplo bem-sucedido de prototipagem de baixo custo. SIMPEP: XXXIV SIMPEP-SIMPÓSIO DE ENGENHARIA DE PRODUÇÃO, 1-14.

Onisaki, H. H. C., \& Vieira, R. M.B. (2019). Impressão 3D e o desenvolvimento de produtos educacionais. Revista de Estudos e Pesquisas sobre Ensino Tecnológico (EDUCITEC), 5 (10), 128-137. https://doi.org/10.31417/educitec.v5i10.638

Orlando, T. C. et al. (2009). Planejamento, montagem e aplicação de modelos didáticos para abordagem de biologia celular e molecular no ensino médio por graduandos de ciências biológicas. Revista Brasileira de ensino de bioquímica e biologia molecular, 2 (1), 2-17. https://doi.org/10.16923/reb.v7i1.33

Ponciano, T. M., Gomes, S. V., \& Morais, I.C. (2017). Metodologia ativa na engenharia: verificação da abp em uma disciplina de engenharia de produção e um modelo passo a passo. Revista Principia, 32-39.

Prado, A. N., Mattos, É. C. A., \& Rodrigues, F.S. (2019). Manufatura Aditiva: conceitos, aplicações e impactos na gestão. III EIGEDIM 2019: III Encontro Internacional de Gestão, Desenvolvimento e Inovação, EIGEDIM, 1689-1699.

Saffaro, F. A. (2007). Uso da prototipagem para a gestão do processo de produção na construção civil. Tese de doutorado em à Universidade Federal de Santa Catarina para Doutorado em Engenharia Civil, Universidade Federal De Santa Catarina - UFSC, Santa Catarina.

Santos, K., Loures, E., Piechnicki, F. \& Canciglieri, O. (2017). Opportunities Assessment of Product Development Process in Industry 4.0. Procedia Manufacturing. FAIM2017: 27th International Conference on Flexible Automation and Intelligent Manufacturing, Procedia Manufacturing, Modelana, 11:1358-1365. http://doi: 10.1016/j.promfg.2017.07.265.

Santos, J. T. G., \& Andrade, A. F. (2020). Impressão 3D como Recurso para o Desenvolvimento de Material Didático: Associando a Cultura Maker à Resolução de Problemas", Revista Novas Tecnologias Na Educação, 18 (1), $21-24$.

Valente, J. A., Almeida, M. E. B., \& Geraldini, A. F. S. (2017). Active methodologies: from conceptions to practices in different levels of teaching. Revista Diálogo Educacional, 17 (52), 455-478. http://dx.doi.org/10.7213/1981-416X.17.052.DS07

Woodson, T., Alcantara, J. T., \& Nascimento, M. S. (2019). Is 3D printing an inclusive innovation: An examination of 3D printing in Brazil. Technovation, $80-81,54-62$. 